\title{
A frequency domain test for isotropy in spatial data models
}

\author{
Flavio Santia ${ }^{\mathrm{a}, *}$, Giuseppe Arbia ${ }^{\mathrm{b}}$, Marco Bee $^{\mathrm{a}}$, Giuseppe Espa ${ }^{\mathrm{a}}$ \\ ${ }^{a}$ Department of Economics and Management, University of Trento \\ Via Inama 5, 38122 Trento (TN), Italy \\ ${ }^{b}$ Department of Statistics, Catholic University of the Sacred Heart \\ L.go Francesco Vito 1, 00168 Rome
}

\begin{abstract}
We develop a new methodology for estimating and testing the form of anisotropy of homogeneous spatial processes. We derive a generalised version of the isotropy test proposed by [1] and analyse its properties in various settings. Expanding on this, we propose a new testing procedure in the frequency domain that allows one to estimate and test under mild conditions any form of anisotropy in homogeneous spatial processes. The power of the test is studied by means of Monte Carlo simulations performed both on regularly and irregularly spaced data. Finally, the method is used to analyse the soybean yields in the US.

Keywords: areal data, semiparametric modelling, directional bias, Fourier analysis, anisotropy modelling
\end{abstract}

\section{Introduction}

Spatial econometric analysis of areal data typically assumes that spatial dependence is equally strong in any direction. This can be noticed if we consider, for example, the SAR model [see 2] or the CAR model [see 3], where the spa5 tial autoregressive parameter is unique and independent of the direction of the neighbours.

\footnotetext{
* Corresponding author

Email address: flavio.santi@unitn.it (Flavio Santi)
} 
Such a restriction does not necessarily hold in any situation, as many economic and social phenomena exhibit a directional bias in their interaction mechanisms and in their values. Consider, for example, the North-South change in the economic and social conditions in several countries, or the importance of direction as an explanatory variable for the correlation amongst harvests in contiguous fields, climatic areas, or geographical regions.

The condition where the characteristics of a phenomenon do not depend on the direction is called isotropy. In particular, a stochastic spatial process is said to be isotropic whenever it is stationary with respect to rotations of its index set about the origin [4, 10]. The assumption of isotropy may lead to inconsistent estimates if it is not borne out by data, similarly to what happens when incorrectly assuming other forms of stationarity. This is the main reason why that condition should be formally tested before fitting any kind of isotropic

20 model.

Over the last decades, the problem of testing isotropy of stochastic spatial processes has received some attention, and isotropy tests have been proposed for stochastic surfaces [5], point processes [e.g. 6], lattice data [7], and (regularly or irregularly-spaced) areal data [1. In this paper we focus on models for areal analysis, and in this framework the problem has received limited attention in the econometric literature. In fact, although it is sometimes possible to adapt isotropy tests like those of [6] or [7] to irregularly-spaced areal data, two issues may make such adaptations not suitable.

30 the best criterion for assessing isotropy of a process that has been modelled through a weight matrix. Indeed, most of the times the physical distance has a minor or no role in defining the weight matrix of a spatial model. It follows that, in these cases, an isotropy test based on variograms [such as 6] or other functions 35 of the physical distance may be inconsistent with the modelling approach being adopted.

Second, samples of irregular areal data are often rather small, especially in 
econometric analysis. This implies that asymptotic isotropy tests can seldom be used. This is the case, for instance, of the test proposed by [7]; although that method can be adapted to irregularly-spaced grids, the outcomes are only reliable in large samples.

The test proposed by Arbia, Bee and Espa [1] (hereinafter ABE) has been developed for econometric models, and overcomes both problems just described. Consider the spatial autoregressive model:

$$
\left\{\begin{array}{l}
y=\rho W y+X \beta+\varepsilon \\
\varepsilon \sim \mathcal{N}_{n}\left(0, \sigma^{2} I\right)
\end{array},\right.
$$

defined on a (regular or irregular) two-dimensional grid $\mathcal{G}_{n}$ of $n$ cells, where $W \in \mathbb{R}^{n \times n}$ is a spatial weight matrix, $X \in \mathbb{R}^{n \times p}$ is a matrix of $p$ exogenous explanatory variables (possibly including a unitary column), $\beta \in \mathbb{R}^{p}, \sigma \in \mathbb{R}^{+}$, 45 and $\rho \in \mathbb{R}$ is such that $I-\rho W$ is positive definite 8 .

The ABE test requires to divide the neighbours of each cell of $\mathcal{G}_{n}$ into two groups according to a unique reference direction $\psi$ and to fit the model:

$$
\left\{\begin{array}{l}
y=\left(\rho_{1} W_{1}+\rho_{2} W_{2}\right) y+X \beta+\varepsilon \\
\varepsilon \sim \mathcal{N}_{n}\left(0, \sigma^{2} I\right)
\end{array} .\right.
$$

where $W_{1}, W_{2} \in \mathbb{R}^{n \times n}$ are the weight matrices that identify the two groups of neighbours, and $\rho_{1}, \rho_{2}$ are the spatial autoregressive parameters.

In the rest of the paper we consider $\tan \psi$ as the slope of the straight line which passes through the centre of any cell $k \in \mathcal{G}_{n}$, splits the plane in two parts, and divides the neighbours of $k$ into two groups. It follows that $\psi$ is the size of the angle between the straight line and the axis of abscissa. Hereinafter we will refer to $\psi$ as reference direction, since it uniquely identifies the related straight line.

Since the groups of neighbours are exclusively defined according to a directional criterion, under isotropy they are expected to have the same effect on the reference cell. It follows that the isotropy assumption holds if $\rho_{1}=\rho_{2}$, and this is the restriction that the $\mathrm{ABE}$ approach formally tests. 
The ABE test works well in many cases, but it may fail to detect some forms of anisotropies, as the following example shows.

Example 1. Let $\left\{y_{r, u}\right\}$ be a spatial process defined on a square lattice $\mathcal{G}_{n}$, and indexed on $\mathbb{Z}^{2}$ such that $y_{r, u}$ is the value of the process $\left\{y_{r, u}\right\}$ in the $r$-th row and the $u$-th column of $\mathcal{G}_{n}$. According to the matrix indexation, rows are numbered from top to bottom whilst columns are numbered from left to right.

Assume that $\left\{y_{r, u}\right\}$ is defined as follows:

$$
y_{r, u}=\gamma_{1}\left(y_{r-1, u}+y_{r, u+1}\right)+\gamma_{2}\left(y_{r+1, u}+y_{r, u-1}\right)+\varepsilon_{r, u},
$$

where $\gamma_{1} \neq \gamma_{2}$ are real parameters and $\left\{\varepsilon_{r, u}\right\}$ is an iid spatial process. The process (3) is clearly anisotropic (since $\gamma_{1} \neq \gamma_{2}$ ).

If we test for isotropy by means of the $\mathrm{ABE}$ test, we may split the neighbours along the NW-SE direction $(\psi=3 \pi / 4)$ and fit the model

$$
y_{r, u}=\rho_{1}\left(y_{r-1, u}+y_{r, u+1}\right)+\rho_{2}\left(y_{r+1, u}+y_{r, u-1}\right)+\varepsilon_{r, u}^{(1)} .
$$

For this specification of the test, model (4) coincides with (3), and it is possible to detect anisotropy provided that the sample size is large enough.

Now assume that we split the neighbours along a different direction. For instance, we may choose the SW-NE direction $(\psi=\pi / 4)$, and fit the model

$$
y_{r, u}=\rho_{1}\left(y_{r-1, u}+y_{r, u-1}\right)+\rho_{2}\left(y_{r+1, u}+y_{r, u+1}\right)+\varepsilon_{r, u}^{(2)} .
$$

Both (4) and (5) are consistent with the ABE method. However, if model (5) is fitted, the anisotropy cannot be detected. This happens because both $\rho_{1}$ and $\rho_{2}$ measure the spatial dependence originating from half-planes including a neighbouring cell with coefficient $\gamma_{1}$ and another neighbour with coefficient $\gamma_{2}$.

Note that model (4) results from any reference direction $\psi$ such that $\pi / 2<$ $\psi<\pi$, whilst model (5) is consistent with any $0<\psi<\pi / 2$.

Example 1 reveals two problems. First, the ABE test is not rotationinvariant, that is, its outcome depends on the direction $\psi$ chosen for splitting the neighbours. Second, there may be compensations between the coefficients 
of the neighbours belonging to the same half-plane. Both issues may cause a substantial reduction of the power of the test.

Having introduced the basic version of the ABE test, we can now outline the main contributions of this paper. First, we extend the ABE test to a generic number $q$ of groups of neighbours (this will be called the $q$-directional ABE test, or simply $q$-ABE test), studying the factors affecting its power and the kind of anisotropies it is able to detect.

Second, building on this analysis, we propose a new approach that allows one to estimate and test the form of anisotropy of any given spatial process without incurring the problems outlined above. This method leads to a semiparametric strategy for estimating and testing spatial anisotropy, based on a Fourier expansion of the function that describes the directional dependence. Unlike the $q$-directional ABE test, this technique does not suffer from mul90 ticollinearity problems when a fine estimation of the directional dependence function is required. The approach is flexible and can be easily applied to models for areal data like spatial autoregressive, spatial error, spatial autoregressive moving average, conditional autoregressive models, etc. Moreover, many forms of anisotropy can be detected by estimating a small number of parameters. Last but not least, the shape, the intensity and the direction of the anisotropies can be estimated.

The paper is organized as follows. Section 2 develops a generalization of the ABE test, focussing on the kind of anisotropies that it can detect, and studying its power. Section 3 introduces the new methodology and illustrates how the form of anisotropy can be estimated and tested on both regularly and irregularly spaced data. Section 4 illustrates the outcomes of Monte Carlo simulation experiments aimed at assessing the finite-sample properties of the estimators and the power of the test. In Section 5 the method is used to analyse the soybean yields in 1430 US counties. Section 6 concludes. 


\subsection{Generalisation of the ABE test to $q$ directions}

The basic ABE test requires the unrestricted model (2) to be tested against the restricted model (1). The generalisation of this approach to any number $q \geq 2$ of directions is straightforward, since we can define the $q$-directional ABE test as a coefficient restriction test for model

$$
y=\left(\sum_{r=1}^{q} \rho_{r} W_{r}\right) y+\varepsilon
$$

which should be compared to

$$
y=\rho\left(\sum_{r=1}^{q} W_{r}\right) y+\varepsilon,
$$

where $\rho_{r}(r=1, \ldots, q)$ are the autoregressive directional parameters and $W_{r}(r=$ $1, \ldots, q)$ are the directional matrices.

Unlike the basic ABE test, the space around each cell is divided into $q$ sectors which correspond to $q$ portions of the round angle, and can be identified by means of intervals of the form

$$
I_{r} \equiv\left[\psi+\frac{2 \pi}{q}(r-1), \quad \psi+\frac{2 \pi}{q} r\right),
$$

where $r=1, \ldots, q$ indexes each sector, and $\psi \in \mathbb{R}$ is the reference direction.

It follows that a neighbour $h$ with Cartesian coordinates $c_{h} \in \mathbb{R}^{2}$ of a cell $k$ with coordinates $c_{k} \in \mathbb{R}^{2}$ belongs to the $r$-th sector if the angle $\psi_{k h}$ between the vector $c_{h}-c_{k}$ and the vector $[1,0]^{\mathrm{T}}$ (that is, the $x$-axis) belongs to the $r$-th interval $I_{r}$. This implies that the elements of the $r$-th directional matrix $(r=1, \ldots, q)$ are defined as follows:

$$
\left(W_{r}\right)_{k h} \equiv \mathbb{1}_{\left\{\psi_{k h} \in I_{r}\right\}} w_{k h}
$$

where $w_{k h}$ is the $(k, h)$ element of $W$ and $\mathbb{1}_{\{\cdot\}}$ is the indicator function.

The following properties of directional matrices follow from (8):

$$
\left\{\begin{array}{l}
\sum_{r=1}^{q} W_{r}=W, \\
W_{r} \odot W_{s}=0 \in \mathbb{R}^{n \times n}, \quad \forall(r, s) \in\{1, \ldots, q\}^{2}: r \neq s,
\end{array}\right.
$$


where $\odot$ is the element-wise matrix product (also known as Hadamard product, see e.g. (9]). The former implies that model (6) reduces to (7) when the isotropy assumption holds (the models are nested); the latter states that each neighbour of any cell can belong to only one of the $q$ sectors.

\subsection{Power of the $q-A B E$ test}

Consider the following generalisation of the spatial process (6):

$$
y_{k}=\sum_{h=1}^{n} f\left(\psi_{k h}\right) w_{k h} y_{h}+\varepsilon_{k},
$$

where $f: \mathbb{R} \mapsto \mathbb{R}$ is bounded, has period $2 \pi$ and is expandable as a Fourier series. Note that we do not make any assumption about the fundamental (that is, the minimum-frequency) period of $f$ : we only require that $f(\omega+2 \pi)=f(\omega)$ for any $\omega \in \mathbb{R}$. Hence, there may exist a positive constant $T<2 \pi$ such that $f(\omega+T)=f(\omega)$ for any $\omega \in \mathbb{R}$. Hereinafter $f$ is referred to as anisotropy function.

With this notation, model $(9)$ is isotropic if $f\left(\psi_{k h}\right)$ does not depend on $\psi_{k h}$ i.e. if $f\left(\psi_{k h}\right)$ is constant, whereas it corresponds to model [6] if $f(\omega)=\rho_{Q(\omega)}$, being $Q: \mathbb{R} \mapsto\{1, \ldots, q\}$ the function that maps angles $\omega \in \mathbb{R}$ to the sector 125 index $\{1, \ldots, q\}]^{1}$ It follows that the functional norm $\left\|f-c_{0}\right\|_{2}^{2}$ with $c_{0} \equiv$ $\frac{1}{2 \pi} \int_{0}^{2 \pi} f(\omega) \mathrm{d} \omega$ can be used as a measure of anisotropy, since $\left\|f-c_{0}\right\|_{2} \geq 0$ for any square-integrable function $f$, whereas $\left\|f-c_{0}\right\|_{2}=0$ only if $f(\omega)=c_{0}$.

With this notation, (9) corresponds to (6) if $f(\omega)=\rho_{Q(\omega)}$, where $Q: \mathbb{R} \mapsto$ $\{1, \ldots, q\}$ is the function that maps angles $\omega \in \mathbb{R}$ to the sector index $\{1, \ldots, q\} \bigsqcup^{2}$

\footnotetext{
${ }^{1}$ In particular, given an angle $\omega$, the function $Q$ returns the index of the circular sector where $\omega$ is located, provided that there are $q$ sectors with amplitude $2 \pi / q$ and sector number one is $[\psi, \psi+2 \pi / q)$. It can be proved that $Q$ is defined as:

$$
Q(\omega)=\left\lfloor q\left(\frac{\omega-\psi}{2 \pi}-\left\lfloor\frac{\omega-\psi}{2 \pi}\right\rfloor\right)+1\right\rfloor
$$

where $\lfloor\cdot\rfloor$ is the floor function, and $\psi$ is the reference direction of the $q$-ABE approximation.

${ }^{2}$ In particular, given an angle $\omega$, the function $Q$ returns the index of the circular sector where $\omega$ is located, provided that there are $q$ sectors with amplitude $2 \pi / q$ and sector number
} 
stant. It follows that the functional norm $\left\|f-c_{0}\right\|_{2}^{2}$ with $c_{0} \equiv \frac{1}{2 \pi} \int_{0}^{2 \pi} f(\omega) \mathrm{d} \omega$ can be used as a measure of anisotropy, since $\left\|f-c_{0}\right\|_{2} \geq 0$ for any square-integrable function $f$, whereas $\left\|f-c_{0}\right\|_{2}=0$ only if $f(\omega)=c_{0}$.

The functional form $f$ in $(9)$ is typically unknown, and the isotropy test aims 135 at testing whether $f$ is constant (isotropy) or not (anisotropy). Nevertheless, when the $q$-ABE test is performed, the process $(9)$ is approximated by means of $(6)$, and then the restriction $\rho_{1}=\rho_{2}=\cdots=\rho_{q}$ is tested. It follows that the hypothesis of isotropy is actually tested for the approximation $\tilde{f}_{q, \psi}$ instead of $f$, as $\tilde{f}_{q, \psi}(\omega)=\rho_{Q(\omega)}$.

If we note that:

$$
\rho_{r}=\frac{q}{2 \pi} \int_{I_{r}} f(\omega) \mathrm{d} \omega
$$

for any $r \in\{1, \ldots, q\}$, the properties of least squares guarantee that:

$$
\left\|\tilde{f}_{q, \psi}-c_{0}\right\|_{2}^{2}=\left\|f-c_{0}\right\|_{2}^{2}-\left\|f-\tilde{f}_{q, \psi}\right\|_{2}^{2}
$$

and:

$$
c_{0}=\frac{1}{2 \pi} \int_{0}^{2 \pi} \tilde{f}_{q, \psi}(\omega) \mathrm{d} \omega=\frac{1}{2 \pi} \int_{0}^{2 \pi} f(\omega) \mathrm{d} \omega .
$$

From Equation (11) it follows that the degree of anisotropy of $f$ is attenuated by the $q$-ABE approximation $\tilde{f}_{q, \psi}$, as $\left\|\tilde{f}_{q, \psi}-c_{0}\right\|_{2}<\left\|f-c_{0}\right\|_{2}$ unless $f=\tilde{f}_{q, \psi}$, and such a reduction gets larger as the approximation $\tilde{f}_{q, \psi}$ of $f$ worsens. This affects the power of the $q$-ABE test, because a small degree of anisotropy in $\tilde{f}_{q, \psi}$ (that is, a small $\left\|\tilde{f}_{q, \psi}-c_{0}\right\|_{2}$ ) makes it more difficult to reject the null ${ }_{145}$ hypothesis that $\tilde{f}_{q, \psi}$ is constant (that is, that $\left\|\tilde{f}_{q, \psi}-c_{0}\right\|_{2}=0$ ) even if $f$ is markedly anisotropic ( $\left\|f-c_{0}\right\|_{2}$ is large).

Hence, for a given $f$, it is possible to improve the power of the $q$-ABE test by setting a number of directions $q$ and a reference angle $\psi$ that reduce the

one is $[\psi, \psi+2 \pi / q)$. It can be proved that $Q$ is defined as:

$$
Q(\omega)=\left\lfloor q\left(\frac{\omega-\psi}{2 \pi}-\left\lfloor\frac{\omega-\psi}{2 \pi}\right\rfloor\right)+1\right\rfloor,
$$

where $\lfloor\cdot\rfloor$ is the floor function, and $\psi$ is the reference direction of the $q$-ABE approximation. 
approximation error $\left\|f-\tilde{f}_{q, \psi}\right\|_{2}$, provided that the effect of the improvement is not neutralised by the reduction in the degrees of freedom due to the increase in the number $q$ of parameters $\rho_{1}, \ldots \rho_{q}$.

Theorem 1 provides some results about the effect of $q$ and $\psi$ on the squared approximation error $\left\|\tilde{f}_{q, \psi}-f\right\|_{2}^{2}$. Hereinafter, real functions $g: \mathbb{R} \rightarrow \mathbb{R}$ in the form $g(\omega)=z \mathrm{e}^{\mathrm{i} n \omega}+\bar{z} \mathrm{e}^{-\mathrm{i} n \omega}$ (with $\bar{z} \in \mathbb{C}$ complex conjugate of $z \in \mathbb{C}$, and $n \in \mathbb{Z})$, or equivalently $g(\omega)=a \cos (n \omega)+b \sin (n \omega)($ with $a, b \in \mathbb{R}$ and $n \in \mathbb{Z})$ are refferred to as harmonics.

Theorem 1. Given a bounded square-integrable anisotropy function $f: \mathbb{R} \rightarrow \mathbb{R}$ with period $2 \pi$ and its approximation $\tilde{f}_{q, \psi}$ based on a $q$-ABE model with reference angle $\psi$, that such that their Fourier expansions are:

$$
f(\omega)=\sum_{n=-\infty}^{\infty} c_{n} \mathrm{e}^{\mathrm{i} n \omega}, \quad \tilde{f}_{q, \psi}(\omega)=\sum_{n=-\infty}^{\infty} \tilde{c}_{n} \mathrm{e}^{\mathrm{i} n \omega},
$$

with $c_{n}, \tilde{c}_{n} \in \mathbb{C}$, then:

(a) the contribution of the $n$-th harmonic to the anisotropy of $f$ in terms of squared norm $\left\|f-c_{0}\right\|_{2}^{2}$ is $\left|c_{n}\right|^{2}+\left|c_{-n}\right|^{2}$;

(b) the contribution of the $n$-th harmonic to the squared approximation error $\left\|\tilde{f}_{q, \psi}-f\right\|_{2}^{2}$ is $\left|\tilde{c}_{n}-c_{n}\right|^{2}+\left|\tilde{c}_{-n}-c_{-n}\right|^{2} ;$

(c) for any non-zero integer $n$ (that is, $n \in \mathbb{Z}^{*}$ ):

$$
\tilde{c}_{n}=\frac{\mathrm{i}}{2 \pi n} \sum_{r=1}^{q}\left[\mathrm{e}^{-\mathrm{i} n\left(\theta+\frac{2 \pi}{q} r\right)}\left(1-\mathrm{e}^{\mathrm{i} n \frac{2 \pi}{q}}\right) \frac{q}{2 \pi} \int_{\theta+\frac{2 \pi}{q}(r-1)}^{\theta+\frac{2 \pi}{q} r} f(s) d s\right] ;
$$

(d) $\lim _{n \rightarrow \pm \infty} \tilde{c}_{n}=0 \quad \forall \psi \in[0,2 \pi)$;

(e) $\lim _{q \rightarrow \infty} \tilde{c}_{n}=c_{n} \quad \forall \psi \in[0,2 \pi)$;

(f) $\tilde{c}_{n}=0$ when $n=k q$ with $k \in \mathbb{Z}^{*}$.

(g) for any pair $\left(q_{0}, \psi_{0}\right)$, it is always possible to construct a sequence of $q$ ABE approximations with parameters $\left\{\left(q_{n}, \psi_{n}\right)\right\}$ such that $\tilde{f}_{q_{n}, \psi_{n}} \stackrel{\mathcal{L}^{2}}{\longrightarrow} f$ as $n \rightarrow \infty$;

(h) the effect of the reference direction $\psi$ on $\left|\tilde{c}_{n}-c_{n}\right|^{2}$ is null when $n=k q$ (for any $k \in \mathbb{Z})$, it tends to be maximum over $[k q,(k+1) q]$ for $n \approx(k+1 / 2) q$ (for any $k \in \mathbb{Z}$ ), and it decreases as $n / q$ diverges; 
(i) the $q-A B E$ approximation with the smallest number of directions $q$ such that it does not compensate positive and negative values of the $n$-th harmonic has parameters $q=2 n$ and $\psi=\operatorname{Arg}\left(c_{n}\right)$ (where $\operatorname{Arg}$ is the principal argument of $c_{n} ;$ see e.g. [10, ch. 1]);

\footnotetext{
${ }^{3}$ It is worth noting that the condition $q \geq 2 n$ (Point $i$ ) identifies the bound defined by the Nyquist frequency [see e.g. 11], as formalised by the Nyquist-Shannon theorem of signal theory [see e.g. 12 .

${ }^{4}$ This value resulted from Monte Carlo simulations whose details are available upon request.
} 
$(q, \psi)$ having a single harmonic as a reference. In the following we call this harmonic the reference harmonic.

To identify the reference harmonic, we assume that the spectrum of $f$ (that is, the sequence of Fourier coefficients $\left\{c_{n}\right\}$ ) is known. In such a case, it is 200 possible to identify the harmonics with the largest value of $\left|c_{n}\right|^{2}+\left|c_{-n}\right|^{2}$ and to choose amongst them the one with the highest frequency, which can be taken as the reference harmonic and used to determine $q$ according to Theorem 1

Obviously, $f$ is and its spectrum are unknown, hence the reference harmonic cannot be easily identified, but two facts are helpful. First, the $q$-ABE approximation implies that, if the reference harmonic and the needed accuracy suggest to choose $q=m$, and a $\tilde{q}$-ABE approximation with $\tilde{q}>m$ is fitted, the results are still reliable and useful. Nevertheless, there is a reduction of degrees of freedom and an increase of multicollinearity.

Second, the setup itself should suggest the maximum frequency of relevant harmonics (that is, the frequency of the reference harmonic), which should allow one to determine a reasonable value for $q$. Consider, for example, data on wheat trials [see e.g. 13], where eight directions are probably enough in order capture the relevant anisotropy originating along the four cadinal directions (North, South, West, East); in this a case the harmonic with angular frequency $n=8$ 215 may be assumed as the reference harmonic.

Once $q$ has been set, a suitable value for $\psi$ needs to be found. Unfortunately, its optimal value depends on the unknown function $f$. This problem can be tackled in two ways: by choosing a larger $q$, or by fitting several $q$-ABE models with various $\psi \in[0,2 \pi / q)$ and choosing the result with the highest $p$-value as the ultimate $\psi$. The former solution relies on Point (e) of Theorem 1, but may lead to a substantial absorption of degrees of freedom and to an unbearable multicollinearity. Moreover, there may be some computational difficulties in handling a large number of directional matrices $\left(W_{1}, \ldots, W_{q} \in \mathbb{R}^{n \times n}\right)$ if the sample size $n$ is large. The latter solution follows from Point (h) of Theorem 1.

225 but may be unfeasible because of computational constraints deriving from fitting several $q$-ABE models. 
Two more remarks about Theorem 1 are worth of consideration. First, the practical meaning of Points (d) and (e) has to do with the ability of the $q$-ABE test in capturing the anisotropy originating from a given harmonic. In particular, Point (d) implies that high-frequency components of $f$ (that is, those with angular frequency $n \gg q$ ) are poorly approximated by $\tilde{f}_{q, \psi}$, regardless of the value of the reference angle $\psi$. On the other hand, Point (e) implies that the low-frequency components of $f$ (those with angular frequency $n \ll q$ ) are well approximated by $\tilde{f}_{q, \psi}$, whatever is the value of the reference angle $\psi$. Second, the aforementioned multicollinearity arising when $q$ grows stems form the fact that the quantities $W_{r} y$ (fot $r=1, \ldots, q$ ) in (6) are increasingly correlated, and this happens when $f$ is nearly constant over several intervals in $[0,2 \pi)$. More precisely, it can be shown that the multicollinearity amongst $W_{r} y$ (for $r=1, \ldots, q)$ increases when the probability mass of the random variable $f(U)$, with $U \sim \mathcal{U}(0,2 \pi)$, concentrates on a finite number $p$ of points and $q$ exceeds $p$.

\section{A new isotropy test}

The problem of finding the optimal values of $q$ and $\psi$ is bypassed by the isotropy test we are going to propose in this section. This new test may be interpreted as a $\infty$-directional $\mathrm{ABE}$ test where the reference angle $\psi$ is no longer necessary, and one estimates the Fourier coefficients of $f$ instead of the directional autoregressive parameters $\rho_{r} \mathrm{~s}$. In the following we will refer to the new test as to the FD-test, as it is developed in the frequency domain.

The difficulties discussed in the previous section arise from the discretisation of the interval $[0,2 \pi)$, and the related necessity of defining two partitioning parameters $(q$ and $\psi)$ and integrating the function $f$. These drawbacks of the $q$-directional ABE test are wiped out when $q \rightarrow \infty$.

When $q \rightarrow \infty$, there is an infinite number of autoregressive parameters $\rho_{r} \mathrm{~s}$, whose estimation is therefore impossible. However, it is possible to estimate the Fourier expansion of $f$ : although the Fourier coefficients are infinite too, 
the harmonic with angular frequency $m$ is fully identified by only two Fourier coefficients $\left(c_{n}\right.$ and $\left.c_{-n}\right)$, while the $q$-ABE test requires $q \geq 2 m$ (Point $i$ of Theorem 11), that is, at least $2 m$ coefficients have to be estimated in order to detect the same harmonic.

A further advantage of the FD-test is related to the orthogonality of the harmonics in a Fourier series. This property entails that the accuracy of the approximation of $f$ can be improved without increasing multicollinearity amongst the regressors. On the contrary, in the $q$-dimensional ABE test, multicollinearity increases as $q$ gets larger.

The new testing approach requires to restate the Fourier expansion of $f$ in 12 as follows:

$$
f(\omega)=\rho+\sum_{m=1}^{\infty}\left[\rho_{c m} \cos (m \omega)+\rho_{s m} \sin (m \omega)\right],
$$

where $\rho \equiv c_{0}, \rho_{c m} \equiv\left(c_{n}+c_{-n}\right)$ and $\rho_{s m} \equiv \mathrm{i}\left(c_{n}-c_{-n}\right)$. It follows that (9) can be rewritten as:

$$
\begin{aligned}
y_{k}= & \rho \sum_{h=1}^{n} w_{k h} y_{h}+ \\
& +\sum_{m=1}^{\infty}\left(\rho_{c m} \sum_{h=1}^{n} \cos \left(m \psi_{k h}\right) w_{k h} y_{h}+\rho_{s m} \sum_{h=1}^{n} \sin \left(m \psi_{k h}\right) w_{k h} y_{h}\right)+\varepsilon_{k},
\end{aligned}
$$

or, in matrix notation,

$$
y=\left(\rho W+\sum_{m=1}^{\infty}\left[\rho_{c m} A_{m} \odot W+\rho_{s m} B_{m} \odot W\right]\right) y+\varepsilon,
$$

where $\left(A_{m}\right)_{k h} \equiv \cos \left(m \psi_{k h}\right),\left(B_{m}\right)_{k h} \equiv \sin \left(m \psi_{k h}\right)$, and the matrix of angles $(\Psi)_{k h} \equiv \psi_{k h}$ can be computed as follows:

$$
\Psi=\operatorname{Arg}\left(\iota_{n} z^{\mathrm{T}}-z \iota_{n}^{\mathrm{T}}\right),
$$
argument, whereas $x \in \mathbb{R}^{n}$ and $y \in \mathbb{R}^{n}$ are the components of the Cartesian coordinates of the cell centroids. 
From 15 it follows that the isotropy condition is:

$$
\rho_{c 1}=\rho_{c 2}=\cdots=\rho_{s 1}=\rho_{s 2}=\cdots=0
$$

so that 15 is isotropic when $f$ does not have any harmonic, i.e. it is constant. If, in addition to (16), we require that $\rho=0$, we can test the hypothesis of no spatial correlation. As is discussed below, specification 15 potentially allows one to model, estimate, and test any form of anisotropy based on any function $f: \mathbb{R} \rightarrow \mathbb{R}$ of period $2 \pi$ expandable in a Fourier series.

As only a finite number of harmonics can be estimated and tested, only some terms of the sum in (15) can be included into the model to be fitted. Nonetheless, 275 a very small number of harmonics can provide an accurate approximation of the functions $f$ relevant for applications, as the high-frequency components of $f$ typically have a marginal role in defining the form of anisotropy. As of this issue, the following example considers a commonly encountered anisotropy structure.

Example 2. The function

$$
f(\omega)=0.1+\alpha e^{-2(\omega-\kappa)^{2}},
$$

plotted in Figure 1 for various values of $\alpha$ and for $\kappa=\pi / 2$, may be suitable for describing the North-South asymmetries existing among regions or administrative units in a country.

As Figure 1 shows, 17 with $\kappa=\pi / 2$ defines a spatial dependence which is uniform in all directions except for the North $(\omega=\pi / 2)$ : as $\omega$ gets closer to $\pi / 2$, the spatial dependence becomes stronger (when $\alpha>0$ ), weaker $(-0.1<\alpha<0$ ), or negative $(\alpha<-0.1)$. The parameters $\alpha$ and $\kappa$ respectively determine the strength and the direction of the anisotropy.

Figure 2 shows the Fourier approximations of $f$ for $\alpha=0.1$ truncated at the second and third term. The function is reasonably well approximated by a Fourier expansion truncated at the second term, as can be seen from the Fourier coefficients of $f$ displayed in Figure 3 the first two harmonics explain most of the variability of $f$, and only a negligible improvement is achieved by including also the third and fourth harmonic. 


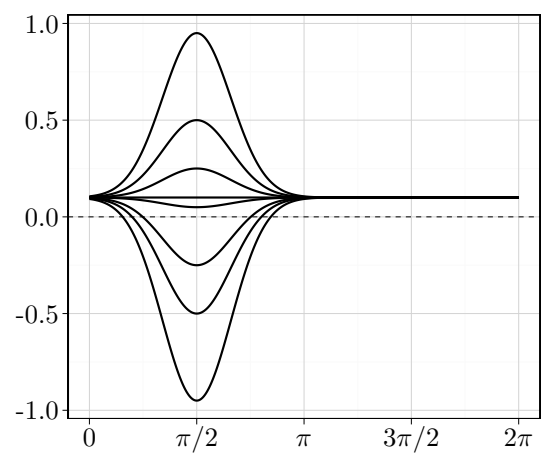

Figure 1: The function 17 for $\kappa=\pi / 2$ and $\alpha$ equal to (from top to bottom) 0.85 , $0.4,0.15,0,-0.05,-0.35,-0.6,-1.05$.

Figure 4 shows the Fourier coefficients of the step function:

$$
f(\omega)=0.1+0.1 \cdot \mathbb{1}_{\{\omega \in(\pi / 4,3 \pi / 4)\}},
$$

whose shape is rather different from (17) with $\kappa=\pi / 2$. It is worth noting that also in this case the first two/three harmonics explain most of the variability of 18 .

The harmonics of $f$ may be given a precise interpretation in terms of the shape of the anisotropy of a process, especially when there are few components. In general, the harmonic with angular frequency $m$ describes a stronger (or weaker) spatial dependence along $m$ directions equally spaced on the round angle. Consider, for example, the harmonic $-\cos (2 \omega)$ illustrated in Figure 5 a In this case the harmonic describes a positive and symmetric spatial dependence along the direction identified by angles $\pi / 2$ (North) and $3 \pi / 2$ (South), and a negative spatial dependence along direction 0 (East) and $\pi$ (West). In other words, the effect of the harmonic $-\cos (2 \omega)$ consists in (symmetrically) increasing the positive spatial dependence along the North-South direction, and decreasing (or making negative) the spatial dependence along the West-East direction. Obviously, the overall form of the anisotropy depends also on the 


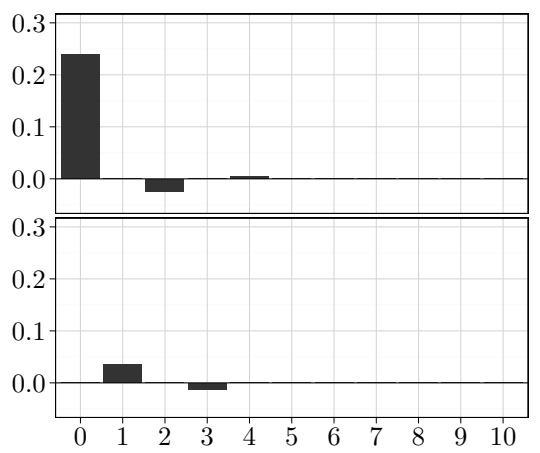

Figure 3: Fourier coefficients $\rho, \rho_{c 1}$, $\ldots, \rho_{c 10}$ (upper panel) and $\rho_{s 1}, \ldots, \rho_{s 10}$ (lower panel) of the function 17 for $\kappa=$ $\pi / 2$ and $\alpha=0.1$.

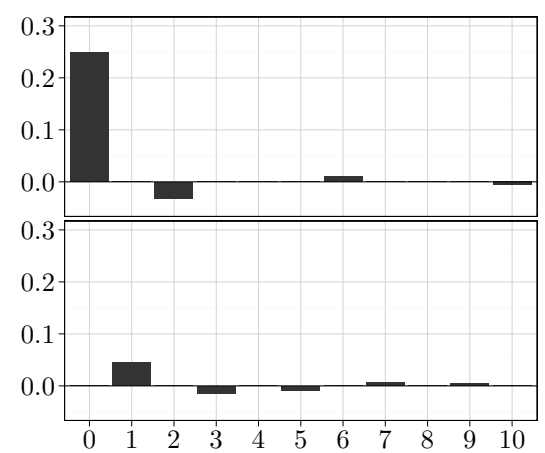

Figure 4: Fourier coefficients $\rho, \rho_{c 1}$, $\ldots, \rho_{c 10}$ (upper panel) and $\rho_{s 1}, \ldots, \rho_{s 10}$ (lower panel) of the function 18 .

other harmonics of $f$, the constant term and their relative amplitudes.

It is possible to describe a relevant class of anisotropies by means of only one or two harmonics, as hinted in Example 2. Figure $5 \mathrm{~b}$ displays the function

$$
f(\omega)=0.1+0.025 \sin \omega-0.05 \cos (2 \omega),
$$

which consists of two harmonics (with angular frequencies $m=1$ and $m=2$

310

West-East direction ( $\omega \approx 0$ and $\omega \approx \pi$ ), while it gets stronger and asymmetric along the North-South direction $(\omega \approx \pi / 2$ and $\omega \approx 3 \pi / 2)$. This shape of $f$ may be useful for describing a spatial dependence that is both stronger (or weaker) and asymmetric along one direction.

The specification of model (15) requires some adaptations when the observations are regularly spaced. If this is the case, the set of values taken by $\psi_{k h}$ is regularly spaced too, and some components of $f$ may be redundant or undersampled [i.e. there is aliasing; see e.g. 11]. If these components of $f$ are not removed, the model is not identifiable from a statistical point of view. The following example discusses this issue when the reference space is a rectangular grid. 


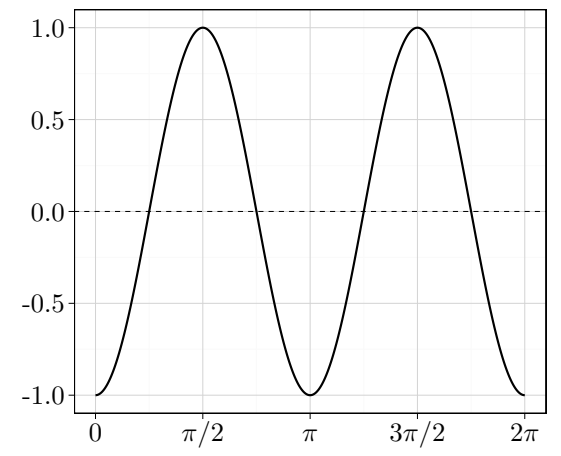

(a) $f(\omega)=-\cos 2 \omega$.

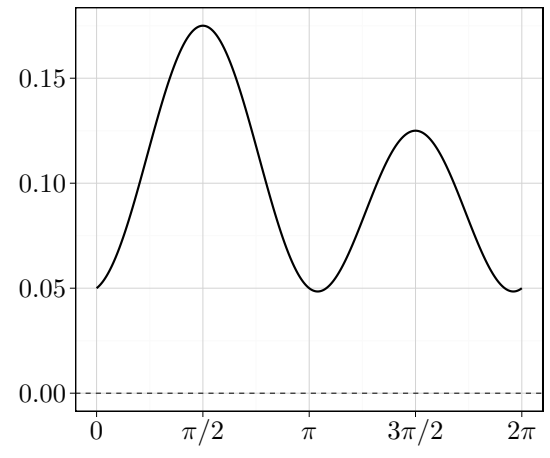

(b) $f(\omega)=0.1+0.025 \sin \omega-0.05 \cos 2 \omega$.

Figure 5: The functions $f$ associated to a single harmonic (Figure 5a), and to a superposition of two harmonics with angular frequencies $m=1$ and $m=2$ (Figure $5 \mathrm{~b}$ ).

Example 3. Consider a square grid $\mathcal{G}_{n}$. We have $\psi_{k h} \in\{0, \pi / 2, \pi, 3 \pi / 2\}$ for all $\psi_{k h} \in \mathcal{G}_{n}$, hence the angular sampling frequency is 4 . In this case, according to the Nyquist-Shannon theorem, only harmonics with angular frequency 1 and 2 should be considered. Moreover, the component $\sin (2 \omega)$ is useless, since $\sin \left(2 \psi_{k h}\right)=0$ for any $(k, h) \in \mathcal{G}_{n}$. Thus, the symmetric anisotropies are captured only by $\cos (2 \omega)$, while the asymmetries originate from $\sin \omega$ (along the North-Sourth direction) and $\cos \omega$ (along the East-West direction). Hence, 15. becomes:

$$
y=\left(\rho W+\rho_{c 1} A_{1} \odot W+\rho_{s 1} B_{1} \odot W+\rho_{c 2} A_{2} \odot W\right) y+\varepsilon .
$$

The test illustrated in this section can be applied to hypotheses different from (16). As pointed out above, the restriction $\rho=0$ along with 16 defines the hypothesis of no spatial correlation. A test based on these two restrictions may be more powerful in detecting spatial dependence than a test for the restriction $\rho=0$ alone. This happens whenever $f$ has the form (14) with $\rho=0$ and some $\rho_{c m}$ or $\rho_{s m}$ different from zero.

In some cases, it may be necessary to test for the presence of a specific form 
of anisotropy, or of asymmetries, or of specific directions in the anisotropy of $f$.

Such formal tests may be interesting in themselves, or may be necessary when the data generating process has to be consistent with certain properties in order to perform further analyses or to apply particular estimation techniques. This is the case, for example, of unilateral approximations [14], which can be used for fitting spatial models defined on a rectangular lattice only if the underlying process is symmetric. Hypotheses of this kind can be easily translated in terms of restrictions on the coefficients of the Fourier expansion of $f$ and tested like any other parameter restriction.

It is worth noting that the possibility of testing specific forms of anisotropy represents a strong advantage of this approach with respect to other isotropy tests such as the $q$-directional $\mathrm{ABE}$ test or the [7] test, which is implicitly based on a specification of 15 truncated at the first term (that is, only the fundamental harmonic with unit angular frequency and the constant term are considered).

In the present section, the reference data generating process is (1), which is referred to as simultaneous autoregressive in the spatial econometric literature [see e.g. 2]. However, our approach can be easily adapted to other econometric models for areal data like spatial error, Durbin, matrix exponential spatial specification [see e.g., 2, 15], conditional autoregressive [see e.g. 16, 3], as well

350 as models based on multiple weight matrices like spatial autocorrelation and spatial autoregressive moving average models [2].

\section{Simulation Study}

In order to study the power of the test in finite samples and compare it to the power of the ABE test, we perform two Monte Carlo simulation experiments on regular and irregular grids. 


\subsection{Regular grid}

We consider a spatial process on a $20 \times 20$ square lattice defined as follows:

$$
\left\{\begin{array}{l}
y=\left(\rho_{1} W_{1}+\rho_{2} W_{2}\right) y+X \beta+u \\
u \sim \mathcal{N}_{n}\left(0, \sigma^{2} I\right)
\end{array},\right.
$$

where $y \in \mathbb{R}^{n}, X \in \mathbb{R}^{n}, \beta=1, \sigma^{2}=1, n=400$. The directional weight matrices $W_{1}$ and $W_{2}$ are defined as $W_{1} \equiv C_{1} \odot W$ and $W_{2} \equiv C_{2} \odot W$, where $C_{1} \in\{0,1\}^{n \times n}$ and $C_{2} \in\{0,1\}^{n \times n}$ are the contiguity matrices of neighbours along the vertical and horizontal direction respectively. The matrix $W \in \mathbb{R}^{n \times n}$ results from a row-standardization of a weight matrix based on the rook neighbourhood rule, and its non-zero elements are independently drawn from the beta distribution $B(2,0.8)$. The regressor $X$ consists of $n$ standard normal random variables.

We employ several values of $\rho_{1}$ and $\rho_{2}\left(\rho_{1}, \rho_{2}=-0.9,-0.8, \ldots, 0, \ldots, 0.8,0.9\right.$ and $\left|\rho_{1}\right|+\left|\rho_{2}\right|<1$; see Table SM.1 of the supplementary material). For each pair $\left(\rho_{1}, \rho_{2}\right)$ we simulate 1000 models and test the isotropy hypothesis $\left(\rho_{1}=\rho_{2}\right)$. All the models share the same directional weight matrices $\left(W_{1}\right.$ and $\left.W_{2}\right)$ and the same regressor $X$. The unrestricted model is based on $f(\omega)=\rho+\rho_{c 2} \cos (2 \omega)$, so that the fitted model is:

$$
y=\rho W y+\rho_{c 2}\left(A_{2} \odot W\right) y+X \beta+\varepsilon .
$$

The isotropy hypothesis $\left(\rho_{c 2}=0\right)$ is tested by means of the likelihood ratio test at a significance level of 5\%. Complete results are shown in Table SM.1 of the supplementary material, while Figure 6 displays the empirical power as a function of $\left|\rho_{1}-\rho_{2}\right|$.

It is worth noting that, in this setting, the 2-ABE test should not be able to detect anisotropy, regardless of the value of $\psi$, because for any $\psi \in[0, \pi)$ there is a perfect compensation amongst coefficients of neighbours belonging to the same half-plane (the condition $n=k q$ with $k=1$ of Theorem 1. Point (f) is verified). It follows that, when $q=2$, anisotropy of model 19 cannot be detected by means of the $\mathrm{ABE}$ test. 


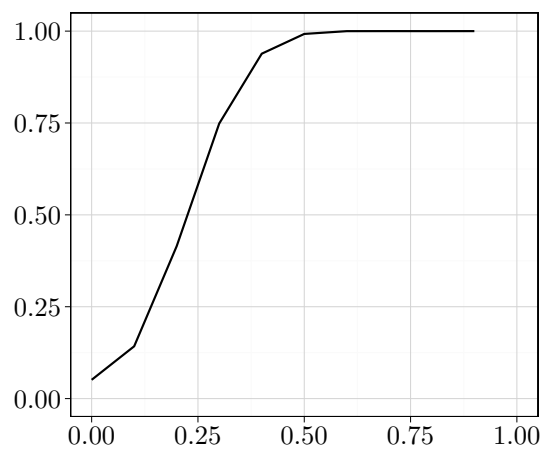

Figure 6: Empirical power of the FD-test (level of significance: $5 \%$ ) for model 19 as a function of $\left|\rho_{1}-\rho_{2}\right|$.

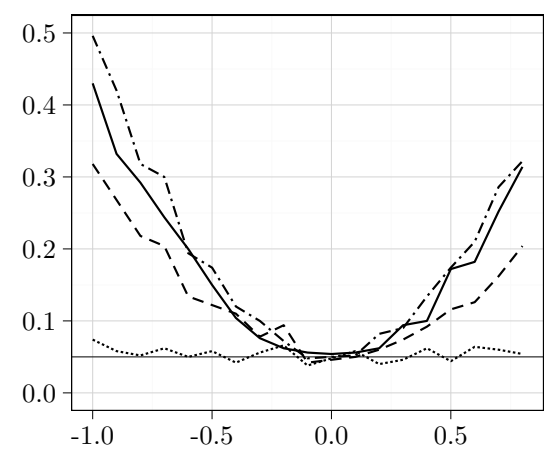

Figure 7: Empirical power of the 2-ABE isotropy test (level of significance: $5 \%$ ) for $\sigma=1$ as a function of $\alpha$ for $\psi=0$ (solid line), $\psi=\pi / 6$ (dot-dashed line), $\psi=\pi / 3$ (dashed line), and $\psi=\pi / 2$ (dotted line).

As Figure 6 clearly shows, the power of the new test sharply increases as the absolute difference between $\rho_{1}$ and $\rho_{2}$ exceeds 0.1 , and it basically equals 1 when $\left|\rho_{1}-\rho_{2}\right|$ is larger than 0.50 .

\subsection{Irregular grid}

The simulations in this section are based on data generating process (9) with the addition of regressors:

$$
y_{k}=\sum_{h=1}^{n} f\left(\psi_{k h}\right) w_{k h} y_{h}+x_{k}^{\mathrm{T}} \beta+\varepsilon_{k} .
$$

We include two regressors with coefficients $\beta=\iota_{2}$, and a regressor matrix $X=$ $\left[\iota_{n}, X_{1}\right] \in \mathbb{R}^{n \times 2}$, where $X_{1}$ is a vector of standard normal random variables. The grid $\mathcal{G}_{n}$ consists of the unit square $[0,1]^{2}$ split into 400 irregular convex polygons obtained by means of a Voronoi tessellation generated by 400 points drawn from the bivariate uniform distribution on $[0,1]^{2}$. The weight matrix $W$ results from row-standardization of a weight matrix $\bar{W}$ built according to the contiguity criterion, and whose non-zero elements equal the areas of the 


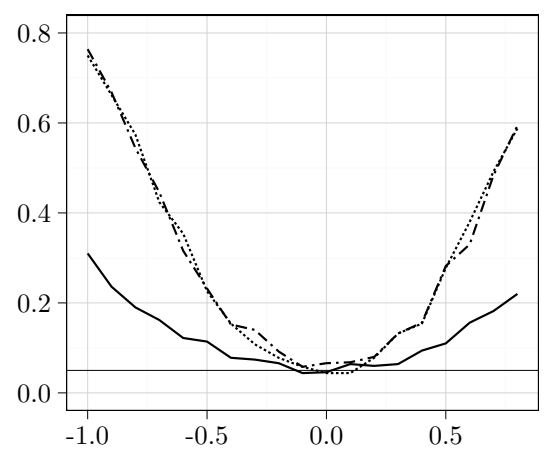

Figure 8: Empirical power of the 4-ABE isotropy test (level of significance: $5 \%$ ) for $\sigma=1$ as a function of $\alpha$ for $\psi=0$ (solid line), $\psi=\pi / 6$ (dot-dashed line), $\psi=\pi / 3$ (dotted line).

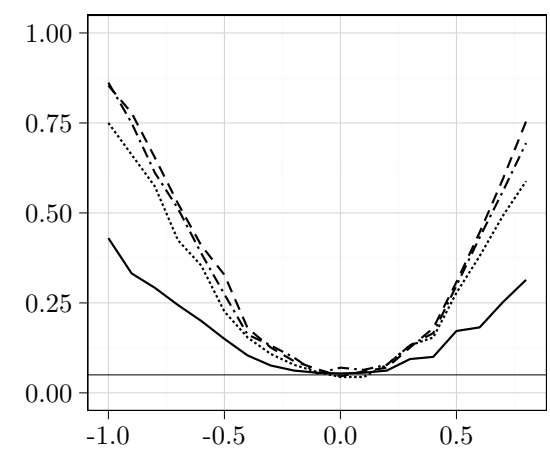

Figure 9: Empirical power of the 2-ABE test with $\psi=0$ (solid line), the $4-\mathrm{ABE}$ test with $\psi=\pi / 3$ (dotted line), the FD test with $\nu=2$ (dashed line), and the FD test with $\nu=3$ (dot-dashed line) for $\sigma=1$ as a function of $\alpha$. All tests are performed at a $5 \%$ significance level.

neighbouring cells. That is, the $(k, h)$ element of $\bar{W}$ is defined as:

$$
(\bar{W})_{k h} \equiv \begin{cases}A_{h} & \text { if } h \text { is a neighbour of } k \\ 0 & \text { otherwise }\end{cases}
$$

where $A_{h}$ is the area of cell $h$.

Hereinafter, as FD-model we consider model (15) with the summation truncated at the $\nu$-th term; that is, only the first $\nu$ harmonics of $f$ are included and fitted. The function $f$ is of type (17), and the simulation has been performed for several values of $\alpha$, provided that the invertibility condition is satisfied, that is $|f(\omega)|<1$ for any $\omega \in[0,2 \pi)$. The innovation $\left\{\varepsilon_{s t}\right\}$ is an iid Gaussian process with standard deviation $\sigma=1,1 / 2,1 / 4$. For each value of $\alpha, \sigma$ and $\nu, 500$ models have been simulated, fitted, and tested by means of the likelihood ratio test, with a significance level equal to $5 \%$. All the models with the same $\alpha, \sigma$ and $\nu$ share the matrix of regressors $X$ and the weight matrix $W$. The grid $\mathcal{G}_{n}$ is shared too. 
The isotropy hypothesis is tested by means of the $2-\mathrm{ABE}$ and the 4 - $\mathrm{ABE}$ test for various values of the reference direction and by means of the FD-test with two and three harmonics ( $\nu=2$ and $\nu=3$ respectively). As for the 2-ABE test, the reference directions $\psi$ should belong to $[0, \pi)$, but we employ four equallyspaced directions in $[0, \pi / 2]$, as the power function is symmetric about $\pi / 2$. In the 4 -ABE test, the reference directions $\psi$ should lie in $[0, \pi / 2)$, and we use three reference directions within this interval. In this case we consider two directions $(\pi / 6$ and $\pi / 3)$, which are symmetrically separated from $\pi / 4$, to check whether the symmetry of the power function is confirmed by the simulation experiments.

Tables SM.2-SM.4 report the complete results, whilst Figures $7 \sqrt{9}$ sum up the most meaningful evidence. According to 10 and the analysis illustrated in Example 1 and in Section 2, given the anisotropy function (17), the power of the $2-\mathrm{ABE}$ test should equal the significance level when $\psi=\kappa$, whilst it reaches the maximum for $\psi=\kappa \pm \pi / 2$.

The outcomes are consistent with the theory (points $h$ and $i$ of Theorem 1), as the empirical power tends to decrease as $\psi$ increases from $0(\kappa-\pi / 2)$ to $\pi / 2$ ( $\kappa$ ), for any value of $\sigma$ (see Figure 7). The power of the 4-ABE test is minimum for $\psi=\kappa \pm \pi / 2$ and maximum for $\psi=\kappa \pm \pi / 4$. Hence, if $\kappa=\pi / 2$, the power is minimum when $\psi=0$, maximum when $\kappa=\pi / 4$, monotonically increasing in $[0, \pi / 4]$, and symmetric about $\psi=\pi / 4$.

The simulation results are consistent with the theoretical results for any $\sigma$, as the empirical power for $\psi=\pi / 6$ and $\psi=\pi / 3$ is higher than the empirical power for $\psi=0$. Moreover, the symmetry is confirmed by the fact that the power of the 4 - $\mathrm{ABE}$ test with $\psi=\pi / 6$ is not statistically different from the empirical power recorded for $\pi / 3$ (see also Figure 8). As expected, the power of the FD-test is higher than the power of the $\mathrm{ABE}$ tests both in case of two and three harmonic specifications (see Figure 9).

The power of the FD-test with two harmonics $(\nu=2)$ is not statistically different from the FD-test specification with three harmonics $(\nu=3)$. In light of Figures 2 and 3 , this is not surprising. 


\section{Application: isotropy in US soybean yields}

The method illustrated in this paper allows one to both test and estimate the form of anisotropy that characterises a given spatial process. The spatial autoregressive model (15) can be generalised by adding a matrix of regressors $X \in \mathbb{R}^{n \times k}$ and restated as:

$$
\left\{\begin{array}{l}
y=\left(F_{\Psi} \odot W\right) y+X \beta+\varepsilon \\
\varepsilon \sim \mathcal{N}_{n}\left(0, \sigma^{2} I\right)
\end{array},\right.
$$

where $\beta \in \mathbb{R}^{k}, \Psi \in \mathbb{R}^{n \times n}$ is the matrix of angles such that $(\Psi)_{k h}=\psi_{k h}$, and $F_{\Psi} \in \mathbb{R}^{n \times n}$ is the matrix with elements $\left(F_{\Psi}\right)_{k h} \equiv f\left(\psi_{k h}\right)$.

It can be verified that:

$$
y \sim \mathcal{N}_{n}\left(\left(I-F_{\Psi} \odot W\right)^{-1} X \beta, \sigma^{2}\left[\left(I-F_{\Psi} \odot W\right)^{\mathrm{T}}\left(I-F_{\Psi} \odot W\right)\right]^{-1}\right) .
$$

A precise and reliable estimation of the correlation matrix is particularly important in agriculture. This issue has been considered in [17, where the corn yields in Iowa between 1926 and 2007 are analysed at a county level. By means of an approach similar to the basic ABE test, the authors find that the spatial dependence is not independent of the direction, and ascertain that this would have a sensible impact on the risk assessment outcomes of the insurance companies.

In this section, we apply the anisotropy test to data on soybean yields for 1430 counties in the United States. The data are provided by the National Agricultural Statistics Service (NASS) of the United States Department of Agriculture (USDA), and result from the survey on soybean yields in 2014. Soybean yields are measured in bushels per acr $5^{5}$ as the ratio between the total produc-

\footnotetext{
${ }^{5}$ The US bushel (symbol bu) is a unit of measure of volumes. However, in case of dry agricultural products, it is used as a measure of weight. For soybean, the United States Department of Agriculture adopts the following conversion for statistical purposes: $1 \mathrm{bu}=27.2 \mathrm{~kg}$. On the other hand, 1 acre (symbol ac) equals $4046.873 \mathrm{~m}^{2}$, so that $1 \mathrm{bu} / \mathrm{ac} \approx 67.212388 \mathrm{~kg} / \mathrm{ha}$. All this information is taken from [18].
} 


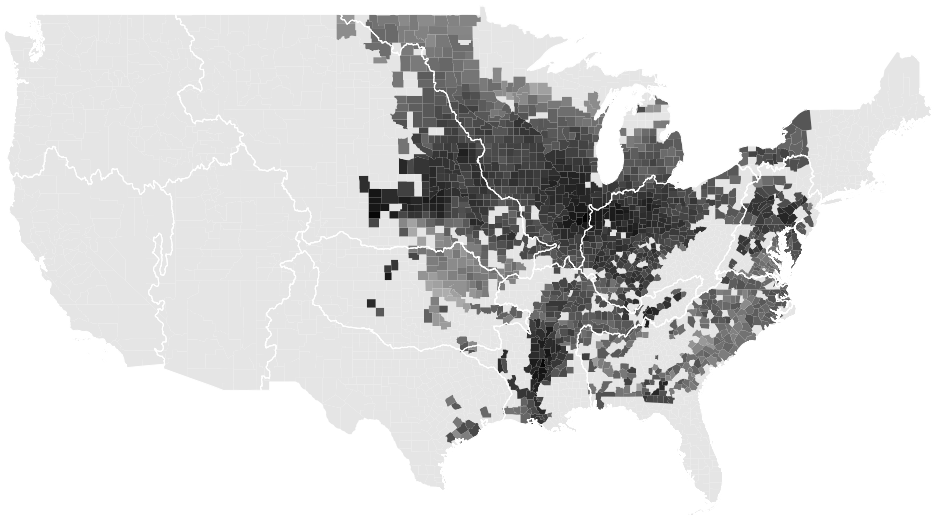

Figure 10: Map of the counties in the Contiguous United States coloured by soybean yields level in 2014 (counties out of the sample are light-gray coloured). The white borders identifies the boundaries of the hydrological basins.

tion in 2014 (first plus successive harvests) and the whole (irrigated and non irrigated) harvested area.

We model the logarithm of the soybean yields by means of the SAR model (21), where the weight matrix $W$ results from a row-standardisation of a contiguous binary matrix $B \in\{0,1\}^{n \times n}$ (where $n=1430$ ). As regressors, we use nine dummy variables for each hydrographic basin where the counties in the sample are located. These variables aim at capturing large-scale spatial effects such as the differences in climate and hydrological conditions of the soil.

Figure 10 shows the location of the 1430 counties along with the boundaries of the hydrographic basins, as determined by the National Operational Hydrologic Remote Sensing Center (NOHRSC) of the National Oceanic and ${ }_{445}$ Atmospheric Administration (NOAA). We assign the counties to hydrographic basins according to the position of their centroids. 


\begin{tabular}{|c|c|c|c|c|c|c|c|}
\hline & $\nu=0$ & $\nu=1$ & $\nu=2$ & $\nu=3$ & $\nu=4$ & $\nu=5$ & $\nu=6$ \\
\hline$\hat{\rho}$ & $\left.\begin{array}{c}0.4194 \\
{[0.0165]} \\
* * *\end{array}\right]$ & $\left.\begin{array}{c}0.4190 \\
{[0.0165]} \\
* * *\end{array}\right]$ & $\left.\begin{array}{c}0.4192 \\
{[0.0165]} \\
* * *\end{array}\right]$ & $\left.\begin{array}{c}0.4200 \\
{[0.0165]} \\
* * *\end{array}\right]$ & $\left.\begin{array}{c}0.4189 \\
{[0.0165]} \\
* * *\end{array}\right]$ & $\left.\begin{array}{c}0.4190 \\
{[0.0164]} \\
* * *\end{array}\right]$ & $\begin{array}{c}0.4186 \\
{[0.0165]} \\
* * *\end{array}$ \\
\hline$\hat{\rho}_{c 1}$ & $\begin{array}{l}- \\
-\end{array}$ & $\begin{array}{c}0.0071 \\
{[0.0041]}\end{array}$ & $\begin{array}{c}0.0072 \\
{[0.0041]}\end{array}$ & $\begin{array}{c}0.0062 \\
{[0.0041]}\end{array}$ & $\begin{array}{c}0.0064 \\
{[0.0041]}\end{array}$ & $\begin{array}{c}0.0065 \\
{[0.0041]}\end{array}$ & $\begin{array}{c}0.0065 \\
{[0.0041]}\end{array}$ \\
\hline$\hat{\rho}_{c 2}$ & - & - & $\begin{array}{l}-0.0030 \\
{[0.0045]}\end{array}$ & $\begin{array}{l}-0.0037 \\
{[0.0045]}\end{array}$ & $\begin{array}{l}-0.0041 \\
{[0.0046]}\end{array}$ & $\begin{array}{l}-0.0041 \\
{[0.0046]}\end{array}$ & $\begin{array}{l}-0.0045 \\
{[0.0046]}\end{array}$ \\
\hline$\hat{\rho}_{c 3}$ & - & $\begin{array}{l}- \\
-\end{array}$ & $\begin{array}{l}- \\
-\end{array}$ & $\begin{array}{c}0.0071 \\
{[0.0046]}\end{array}$ & $\begin{array}{c}0.0071 \\
{[0.0046]}\end{array}$ & $\begin{array}{c}0.0071 \\
{[0.0046]}\end{array}$ & $\begin{array}{c}0.0072 \\
{[0.0046]}\end{array}$ \\
\hline$\hat{\rho}_{c 4}$ & $\begin{array}{l}- \\
-\end{array}$ & $\begin{array}{l}- \\
-\end{array}$ & $\begin{array}{l}- \\
-\end{array}$ & $\begin{array}{l}- \\
-\end{array}$ & $\begin{array}{c}0.0037 \\
{[0.0034]}\end{array}$ & $\begin{array}{c}0.0037 \\
{[0.0034]}\end{array}$ & $\begin{array}{c}0.0037 \\
{[0.0035]}\end{array}$ \\
\hline$\hat{\rho}_{c 5}$ & $\begin{array}{l}- \\
-\end{array}$ & - & $\begin{array}{l}- \\
-\end{array}$ & - & $\begin{array}{l}- \\
-\end{array}$ & $\begin{array}{c}0.0029 \\
{[0.0033]}\end{array}$ & $\begin{array}{c}0.0029 \\
{[0.0033]}\end{array}$ \\
\hline$\hat{\rho}_{c 6}$ & - & $\begin{array}{l}- \\
-\end{array}$ & $\begin{array}{l}- \\
-\end{array}$ & $\begin{array}{l}- \\
-\end{array}$ & - & $\begin{array}{l}- \\
-\end{array}$ & $\begin{array}{c}0.0007 \\
{[0.0029]}\end{array}$ \\
\hline$\hat{\rho}_{s 1}$ & $\begin{array}{ll}- \\
-\end{array}$ & $\begin{array}{c}0.0071 \\
{[0.0044]}\end{array}$ & $\begin{array}{c}0.0066 \\
{[0.0044]}\end{array}$ & $\begin{array}{c}0.0055 \\
{[0.0044]}\end{array}$ & $\begin{array}{c}0.0050 \\
{[0.0044]}\end{array}$ & $\left.\begin{array}{c}0.0050 \\
{[0.0044]}\end{array}\right]$ & $\begin{array}{c}0.0054 \\
{[0.0044]}\end{array}$ \\
\hline$\hat{\rho}_{s 2}$ & - & $\begin{array}{l}- \\
-\end{array}$ & $\begin{array}{c}0.0063 \\
{[0.0049]}\end{array}$ & $\begin{array}{c}0.0055 \\
{[0.0049]}\end{array}$ & $\begin{array}{c}0.0049 \\
{[0.0049]}\end{array}$ & $\begin{array}{c}0.0048 \\
{[0.0049]}\end{array}$ & $\begin{array}{c}0.0054 \\
{[0.0049]}\end{array}$ \\
\hline$\hat{\rho}_{s 3}$ & - & - & $\begin{array}{l}- \\
-\end{array}$ & $\begin{array}{c}0.0094 \\
{[0.0044]} \\
*\end{array}$ & $\begin{array}{c}0.0093 \\
{\left[\begin{array}{c}0.0044] \\
*\end{array}\right]}\end{array}$ & $\begin{array}{c}0.0090 \\
{\left[\begin{array}{c}0.0045] \\
*\end{array}\right]}\end{array}$ & $\begin{array}{c}0.0089 \\
{\left[\begin{array}{c}0.0045] \\
*\end{array}\right]}\end{array}$ \\
\hline$\hat{\rho}_{s 4}$ & - & - & $\begin{array}{l}- \\
-\end{array}$ & $\begin{array}{l}- \\
-\end{array}$ & $\begin{array}{c}0.0060 \\
{[0.0040]}\end{array}$ & $\begin{array}{c}0.0059 \\
{[0.0040]}\end{array}$ & $\begin{array}{c}0.0066 \\
{[0.0041]}\end{array}$ \\
\hline$\hat{\rho}_{s 5}$ & - & - & - & - & $\begin{array}{l}- \\
-\end{array}$ & $\begin{array}{l}-0.0004 \\
{[0.0032]}\end{array}$ & $\begin{array}{l}-0.0003 \\
{[0.0032]}\end{array}$ \\
\hline$\hat{\rho}_{s 6}$ & - & - & $\begin{array}{l}- \\
-\end{array}$ & - & - & $\begin{array}{l}- \\
-\end{array}$ & $\begin{array}{c}0.0048 \\
{[0.0031]}\end{array}$ \\
\hline loglik & 603.31 & 605.85 & 606.94 & 610.58 & 612.37 & 612.77 & 613.95 \\
\hline$p$-value & - & 0.07899 & 0.12278 & 0.02408 & 0.02033 & 0.04119 & 0.04626 \\
\hline
\end{tabular}

Table 1: Estimated coefficients of the regressions for testing isotropy with various numbers of harmonics $(\nu=0, \ldots 6)$. The table only reports the coefficients of the components of $f$, their standard errors (in brackets), and the level of significance ("." for $p$-values between 0.1 and 0.05 , "** for $p$-values between 0.05 and 0.01 , "**" for $p$-values between 0.01 and 0.001 , "***" for $p$-values smaller than 0.001 , and nothing when the $p$-values are higher than 0.1 ). All the coefficients of the dummy variables have a $p$-value lower than 0.001 for all models. At the bottom of the table we report the maximized log-likelihood of the estimated model and the $p$-value of the anisotropy test.

We carry out the FD isotropy testing procedure by including various numbers of harmonics (from a minimum of one to a maximum of six). The models are fitted via maximum likelhood, whereas parameter restrictions are tested using 
the log-likelihood ratio test; the results are summarized in Table 1. The data have a slight form of anisotropy, significant at the $5 \%$ level. The only singularly significant harmonic at the $5 \%$ level is the third one.

\begin{tabular}{cccl}
\hline$q$ & $\psi$ & log-likelihood & $p$-value \\
\hline 2 & 0 & 606.01 & $0.0202^{*}$ \\
2 & $\pi / 2$ & 604.33 & 0.1521 \\
3 & 0 & 607.53 & $0.0147^{*}$ \\
3 & $\pi / 6$ & 605.05 & 0.1758 \\
3 & $\pi / 3$ & 604.27 & 0.3825 \\
6 & 0 & 609.11 & $0.0406^{*}$ \\
6 & $\pi / 12$ & 605.82 & $0.0808^{\bullet}$ \\
6 & $\pi / 6$ & 606.72 & 0.2349 \\
\hline
\end{tabular}

Table 2: Maximised log-likelihood of $q$-ABE approximations on soybeans yield data for various specifications $(q, \psi)$. The table reports also the $p$-values of the log-likelihood ratio test on the isotropy assumption, and symbols about the level of significance ("." for $p$-values between 0.1 and 0.05 , "**" for $p$-values between 0.05 and 0.01 , "**" for $p$-values between 0.01 and 0.001 , “***" for $p$-values smaller than 0.001 , and nothing when the $p$-values are higher than 0.1 ). All the coefficients of the dummy variables have a $p$-value lower than 0.001 for all models. All fitted models included the same regressors as those of Table 1

The $q$-ABE isotropy test with various specifications $(q, \psi)$ and the same regressors on hydrographic basins has been performed as well; the results are summarised in Table 2, where $p$-values of the log-likelihood ratio test on the isotropy hypothesis are reported. The results in Table 2 confirm the problems of the $q$-ABE test discussed in Section 2, in particular, it is apparent the marked sensitivity of the $p$-value with respect to the reference direction $\psi$ : for any value of $q=2,3,6$ there is a specification of the $q$-ABE test where evidence against the isotropy assumption is found, however, a little variation of $\psi$ may strongly increase the $p$-value of the test, suggesting a clear evidence in favour of the isotropy hypothesis. 


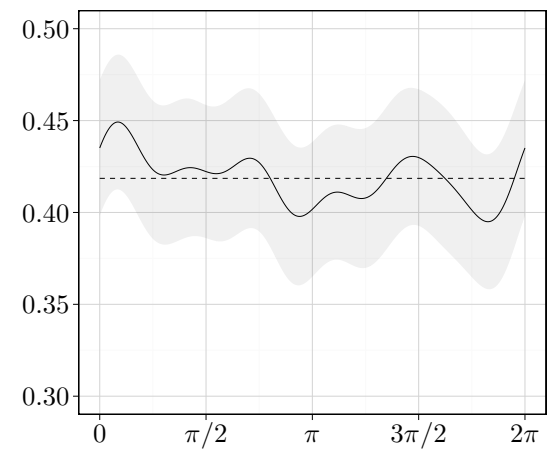

(a)

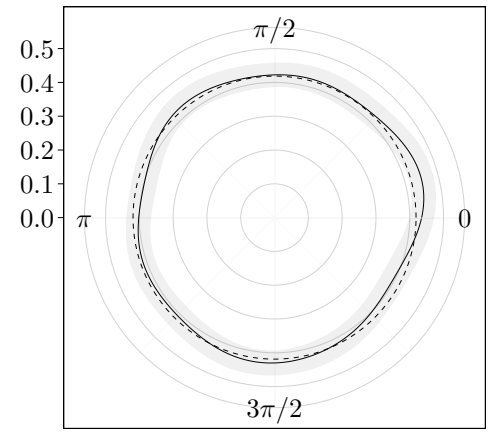

(b)

Figure 11: The function $f$ (solid line) of the SAR model on soybean yields estimated by means of the first six harmonics in Cartesian (Figure 11a) and polar (Figure 11b) coordinates. The shaded area represents the $95 \%$ confidence interval on $f$. The dashed line is the mean value of $f$.

The estimates of the harmonic coefficients in Table 1 are very stable amongst the various specifications of the FD test both in terms of point estimates and standard errors. This results form the orthogonality property of harmonics based on Fourier series, and proves that there is no multi-collinearity amongst the vectors $A_{1} \odot W y, \ldots, A_{\nu} \odot W y, B_{1} \odot W y, \ldots, B_{\nu} \odot W y$ in 15 . This property is particularly important from an applicative point of view, as it permits a reliable estimate of the harmonic coefficients of $f$ included in the fitted model even if some other relevant harmonics are excluded.

The shape of the anisotropy estimated by means of the first six harmonics is plotted in Figures 11a and 11b. It can be noticed that the spatial dependence is slightly stronger along the East-North-East $(\omega \approx \pi / 8)$, North-West $(\omega \approx 3 \pi / 4)$, and South $(\omega \approx 3 \pi / 2)$ directions, whilst it is weaker along the West $(\omega \approx \pi)$, and the South-East $(\omega \approx 7 \pi / 4)$ directions. The function $f$ estimated with a smaller number of harmonics $(\nu<6)$ is rather close to that plotted in Figure 11 . although some peaks are less pronounced.

The analysis suggests that there is anisotropy in the soybean yields in the US 
counties in 2014. Although the directional variation in the spatial dependence is small, this may have a remarkable effect on the systemic risk of a portfolio of insurance policies or derivatives on crop yields. This happens because, according to (22), the anisotropy function $f$ affects the covariance matrix of the crop yield, and correlations amongst assets of a financial portfolio heavily affect the profit and loss distribution, in particular the probability of extremal events [see e.g. 485 19.

\section{Conclusion}

In this paper a generalisation of the $\mathrm{ABE}$ isotropy test [1] is proposed and its theoretical properties are analysed in order to study how its various specifications can affect the power of the test.

The application and theoretical drawbacks of the $q$-ABE test are overcome by the frequency-domain test proposed in this paper, which permits to test and estimate the shape of the anisotropy. The semi-parametric nature of this method makes it applicable to various models for areal data based on weight matrices, while the algebraic properties of the Fourier series minimise the multicollinearity problems possibly originated by an accurate specification of the anisotropy function $f$. The Monte Carlo simulations have shown that the FD test outperform the $q$-ABE test in terms of power. Moreover, the FD test is easier to implement than the $q$-ABE test.

The test relies on the assumption of homogeneity of the data generating process, and this may be, in some cases, a rather strong assumption. The generalisation of the test to non-homogeneous spatial processes is a subject that deserves further research.

\section{SUPPLEMENTARY MATERIAL}

Proofs and complete results of Monte Carlo simulations. Proof of Theregular and irregular grids (see Section 4). (pdf) 


\section{References}

[1] G. Arbia, M. Bee, G. Espa, Testing isotropy in spatial econometric models, Spatial Economic Analysis 8 (3) (2013) 228-240.

[2] J. P. LeSage, R. K. Pace, Introduction to Spatial Econometrics, Chapmann\&Hall, 2009.

[3] N. A. C. Cressie, Statistics for Spatial Data, revised edition Edition, John Wiley \& Sons, 2015.

[4] B. D. Ripley, Spatial Statistics, John Wiley \& Sons, 1981.

515 [5] E. M. Cabaña, Affine processes: A test of isotropy based on level sets, SIAM Journal on Applied Mathematics 47 (4) (1987) 886-891.

[6] Y. Guan, M. Sherman, J. A. Calvin, A nonparametric test for spatial isotropy using subsampling, Journal of the American Statistical Association 99 (467) (2004) 810-821.

[7] A. Molina, F. R. Feito, A method for testing anisotropy and quantifying its direction in digital images, Computer \& Graphics 26 (2002) 771-784.

[8] J. K. Ord, Estimation methods for models of spatial interaction, Journal of the American Statistical Association 70 (349) (1975) 120-126.

[9] R. A. Horn, C. R. Johnson, Matrix Analysis, 2nd Edition, Cambridge University Press, 2013.

[10] T. M. Apostol, Mathematical Analysis, 2nd Edition, Prentice Hall, 1974.

[11] P. Bloomfield, Fourier Analysis of Time Series, 2nd Edition, John Wiley \& Sons, 2000.

[12] R. Priemer, Introductory Signal Processing, World Scientific Publishing, 1991.

[13] P. Whittle, On stationary processes in the plane, Biometrika 41 (3/4) (1954) 434-449. 
[14] G. Arbia, M. Bee, G. Espa, F. Santi, Fitting spatial regressions to large datasets using unilateral approximations, Communications in Statistics

$535 \quad-$ Theory and Methods (forthcoming). doi:10.1080/03610926.2017. 1301476

[15] G. Arbia, A Primer for Spatial Econometrics, Palgrave, 2014.

[16] M. M. Wall, A close look at the spatial structure implied by the CAR and SAR models, Journal of Statistical Planning and Inference 121 (2004) 311-324.

[17] Y. Zhu, S. K. Ghosh, B. K. Goodwin, Directional spatial dependence and its implications for modeling systemic yield risk, 2009 annual meeting, july 26-28, 2009, milwaukee, wisconsin, Agricultural and Applied Economics Association (2009).

545 [18] Weights and Measures Division Technology Services, The International System of Units (SI) - Conversion Factors for General Use, NIST Special Publication 1038, National Institute for Standard and Technology - U.S. Department of Commerce (May 2006).

[19] A. J. McNeil, R. Frey, P. Embrechts, Quantitative Risk Management, 550 Princeton University Press, 2005. 